\title{
Visual field differences in the magnitude of the Oppel-Kundt illusion vary with processing time
}

\author{
BART ROTHWELL and ERAN ZAIDEL \\ University of California, Los Angeles, California
}

\begin{abstract}
Three experiments were performed to investigate hemispheric differences in susceptibility to the Oppel-Kundt illusion presented tachistoscopically to the two visual hemifields. Experiment 1 used a successive comparison mode, in which 16 undergraduate students indicated whether the second of two successive extents looked shorter or longer than the first. Experiment 2 (20 undergraduates) and Experiment 3 (1 commissurotomy patient) required judgments of two extents presented simultaneously. The first experiment found no significant visual field differences, although females were more susceptible than males. In the second experiment, the illusion magnitude was greater in the left visual field, and in the third experiment, it was greater in the right visual field. Post hoc analyses resolve the conflicting results and show that in all three experiments, the susceptibility of the right hemisphere declined more than that of the left hemisphere during illusion processing. An interpretation is offered in terms of two parallel processes, one that is fast, uses feature extraction, and is performed more effectively in the left hemisphere, and one that is slow, uses visuospatial analysis to compute distances between parts of the illusion figure, and is performed more effectively in the right hemisphere.
\end{abstract}

Although many functional differences between the-cerebral hemispheres have been studied, relatively few attempts have been made to learn about the lateralization of susceptibility to visual illusions. This fact is surprising, because the existence of a hemispheric asymmetry in susceptibility to illusions would have important consequences for existing theories about hemispheric specialization and independence in visual information processing. In addition, knowledge of such an asymmetry would lead to a better overall understanding of how visual illusions are processed.

\section{Predictions from Theory}

Present theories about the processing styles of the two hemispheres lead to conflicting predictions regarding their relative susceptibilities to visual illusions. A great deal of evidence suggests that the right hemisphere uses a global, holistic processing style, whereas the left hemisphere uses a local, analytic style (Bradshaw \& Nettleton, 1981; Zaidel, 1978). In addition, studies have shown that of the two hemispheres, the right hemisphere is more fielddependent (Cohen, Berent, \& Silverman, 1973; Zaidel, 1973 ) and is more likely to perceive the background of a figure (Cronin-Golomb, 1986). Thus, one might expect

This material is based upon work supported under a National Science Foundation Graduate Fellowship to Bart Rothwell, and NIMH RSDA MH00179, NIH Grant NS20187, and a UCLA Biomedical Research Support Grant to Eran Zaidel. The authors thank Steve Hunt for assistance with the computerized implementation of the testing procedure. Address correspondence to Bart Rothwell, who is now at HNC, Inc., 5501 Oberlin Dr., San Diego, CA 92121-1718. the right hemisphere to have the greater susceptibility to visual illusions, because its tendency to process the global aspects of a visual input would make it less able to extract only the relevant features of an illusion figure without regard for the distracting adjacent features that create the illusion.

Another line of reasoning, however, suggests the opposite. It is generally accepted that the right hemisphere is specialized for visuospatial functions (Bradshaw \& Nettleton, 1983; Davidoff, 1982). Furthermore, Coren and Porac (1987) have shown that individuals with superior spatial abilities are less susceptible to some illusions. Thus, it might be expected that the right hemisphere, with its superior spatial ability, would be less susceptible to many illusions. In particular, this conclusion is supported by the finding that the right hemisphere is superior at computing the distances between parts of a figure (Kosslyn, 1987), because the effect of accurate distance computing in an illusion task would be to defeat the illusion.

\section{Previous Findings}

Previous research regarding hemispheric differences in susceptibility to visual illusions has not resolved the issue. A greater right hemisphere susceptibility has been suggested by studies of the Müller-Lyer illusion in splitbrain patients (Kumar, Zaidel, \& Bogen, 1976) and in patients with unilateral brain damage (Houlard, Fraisse, \& Hecaen, 1976; Kumar \& Bogen, 1974). A greater left hemisphere susceptibility, however, is indicated by another study of the Müller-Lyer illusion in brain-damaged patients (Basso, Bisiach, \& Faglioni, 1974). Furthermore, studies with normal subjects have failed to discover visual 
field differences in the magnitudes of the Ponzo illusion (Bertelson \& Morais, 1983), the Poggendorff illusion (Greist \& Grier, 1977), and the rod-and-frame and tilt illusions (Magnussen, Landro, \& Johnsen, 1985).

Finally, a study by Clem and Pollack (1975) exposed a fundamental complication in the issue. In that study, the Müller-Lyer illusion figure was presented tachistoscopically to the two visual fields of normal subjects in two versions. In a successive version, the figure was presented in two exposures, the first containing only the fins of the figure, and the second (after a delay of $500 \mathrm{msec}$ ) containing only the shaft, whereas in a simultaneous version, the entire figure was presented in a single exposure. Clem and Pollack found that the successive version, which presumably emphasizes the sequential processing style of the left hemisphere, produced a stronger illusion in the right visual field, but the simultaneous version, which presumably emphasizes the parallel processing style of the right hemisphere, produced a stronger illusion in the left visual field.

These findings indicate that hemispheric differences in susceptibility to visual illusions are likely to change with variations in the stimulus parameters, and thus it is not surprising that the few previous investigations have failed to produce consistent results. In order to resolve the issue, it is necessary to extend the range of illusions that have been tested, and to investigate the effects of varying the illusion parameters. It is also necessary to use illusion effects that have strong central processing components, because laterality differences are unlikely to exist at peripheral levels (and this fact may explain the negative results of some of the previous studies).

\section{Rationale of the Present Experiments}

The present experiments were carried out to determine if there are hemispheric differences in susceptibility to the Oppel-Kundt illusion, and to investigate the possibility that varying the temporal parameters of the presenta-

Test

(0)

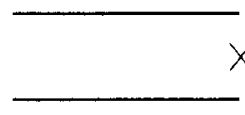

(4)

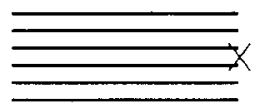

(9)

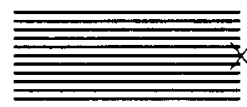

Comparison
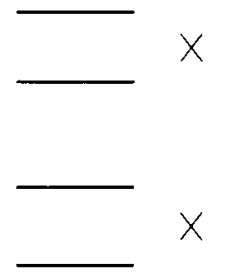

Figure 1. The Oppel-Kundt illusion, demonstrated by the 0,4 , and 9 test figures and the comparison figures used as stimuli in Experiment 1. The three test figures shown here are equal in vertical extent. Crosses show the relative position of fixation for left visual field presentations of these figures.

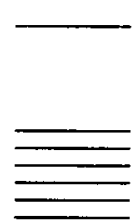

$(20 \%)$

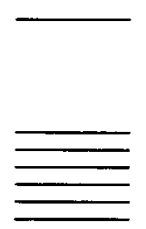

$(30 \%)$
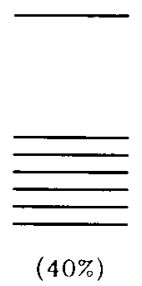

Figure 2. The Oppel-Kundt illusion, demonstrated by the 20\%, $30 \%$, and $40 \%$ figures used as stimuli in Experiment 2 . The percentages are the amounts by which the unfilled extents (top portions) are greater in height than the filled extents (bottom portions). The cross shows the relative position of fixation for a left visual field presentation of the $40 \%$ figure.

tion would affect these differences, as suggested by Clem and Pollack (1975). The Oppel-Kundt illusion, also known as the "illusion of filled extent," is shown in the left column of Figure 1 and in Figure 2 (using stimuli from Experiments 1 and 2, respectively). In this illusion, the extent of a figure is overestimated when there are interposed lines that fill the extent. Thus, in Figure 1, the 4 and 9 test figures (with four and nine interposed lines, respectively) are commonly perceived to be greater in height than the 0 test figure (with no interposed lines, but of the same height). Figure 2 shows three figures in a more compact form of the illusion, in which the unfilled extent (top portion of each figure) is joined with the filled extent (bottom portion). As this figure shows, under certain conditions, the height of the unfilled extent may need to be at least $20 \%$ greater than the height of the filled extent in order for the two heights to be perceived as equal. The Oppel-Kundt illusion was chosen for the present experiments because there is evidence for a central processing component of the illusion effect (Coren \& Hoenig, 1972 , interpreted according to the theory of Girgus \& Coren, 1973), and as noted above, such a component may be necessary for laterality differences to exist. Although the basis for the Oppel-Kundt illusion is not well understood, a difference in attention to the two extents is a possible contributing factor (Piaget, 1961/1969). (For general reviews of the illusion, see Coren \& Girgus, 1978, pp. 26-27, and Robinson, 1972, pp. 49-52.)

In the present experiments, the Oppel-Kundt illusion figures were presented tachistoscopically to the two visual fields of subjects, and hemispheric differences in susceptibility were inferred from the differences between judgments made for the two visual field presentations. In order to obtain a pure measure of the performance of each hemisphere, these judgments were considered valid only when the response hand and all stimuli were contralateral to the hemisphere being tested.

The illusion judgments were made in two modes. In the successive comparison mode (Experiment 1), a test stimulus figure (either a filled or an unfilled extent, as shown in the left column of Figure 1) was followed after 2 sec by a comparison stimulus figure (always an unfilled extent) used as a reference for the judgment of the test figure (see right column of Figure 1). The effect of the 
illusion was then measured by the difference between height judgments of test figures with filled extents and height judgments of test figures with unfilled extents. In the simultaneous comparison mode (Experiment 2, with normal subjects, and Experiment 3, with a split-brain patient), the stimulus figures contained both filled and unfilled extents to be compared with each other (see Figure 2), so that the illusion judgment was made immediately following a single stimulus presentation.

Note that the successive comparison mode of this study differed from the successive presentation version used by Clem and Pollack (1975), in that our successive comparison mode used a delay between the presentation of the full illusion figure and the presentation of the comparison figure, whereas the Clem and Pollack version used a delay between the presentations of two parts that made up the full illusion figure. Thus, the variation between our two comparison modes involved a manipulation of the total illusion processing time, that is, the time spent processing (and remembering) the complete illusion figure before a response was made. Because of this difference from the Clem and Pollack study, and because of the inconclusiveness of previous research, no explicit predictions were made regarding visual field differences in the magnitude of the Oppel-Kundt illusion in either of the two comparison modes.

\section{EXPERIMENT 1 SUCCESSIVE COMPARISONS}

In the first experiment, the Oppel-Kundt illusion was presented in the successive comparison mode, in which subjects were required to process (and remember) the test figure for $2 \mathrm{sec}$ before comparing it to a second figure for the generation of a response. In order to reduce the number of invalid trials (in which the response hand was not ipsilateral to all figures), the subjects were required to respond using the hand on the same side as the visual field of the second figure. Two types of filled extents were used for the test figures (Test Figures 4 and 9 in Figure 1), because the optimal number of interposed lines was not known ahead of time.

\section{Method}

Subjects. Sixteen undergraduate students (8 females and 8 males) participated in partial fulfillment of a course requirement in general psychology at the University of California, Los Angeles. Nine additional subjects participated but were excluded because their data were unreliable (see Design and Procedure subsection). All subjects were strongly right-handed with no familial sinistrality, and all were free of neurological disorders and unusual vision problems, as determined by a questionnaire with handedness items selected from the Edinburgh Handedness Inventory (Bryden, 1977; Oldfield, 1971). The subjects were 16-31 years of age, with a mean age of 19 years, 9 months.

Apparatus. Stimulus presentation and response collection were controlled by an IBM PC-compatible microcomputer. An Amdek Video-310A monochrome (amber) display presented the stimuli against the dark background of the screen, and a chinrest was used to stabilize the viewing distance at $57.3 \mathrm{~cm}$ (so that $1 \mathrm{~cm}$ on the screen represented $1^{\circ}$ of visual angle). Responses were made on a keyboard that was covered so that each hand only had access to two horizontally adjacent keys, labeled $S$ and $L$. The labeling order of these keys was counterbalanced across the subjects. Response times were measured to the nearest millisecond.

Stimuli. The stimuli consisted of a fixation cross, test figures, and comparison figures. The fixation cross was an $\times, 0.5 \times 0.5 \mathrm{~cm}$ in size; it always appeared in the center of the screen, accompanied by a $1000-\mathrm{Hz}$ signal tone for $200 \mathrm{msec}$.

The test figures were of three types, consisting of zero, four, or nine horizontal lines interposed between two outer horizontal lines (Test Figures 0, 4, and 9, respectively, as shown in Figure 1). All of the lines in each test figure were parallel, evenly spaced, $4.0 \mathrm{~cm}$ long, and $0.04 \mathrm{~cm}$ (1 pixel) thick. For each of the three types of test figures, there were 10 different heights, ranging from $0.88 \mathrm{~cm}$ to $4.84 \mathrm{~cm}$ in $0.44-\mathrm{cm}$ increments. Each test figure was centered vertically on fixation and appeared in either the left visual field (LVF) or the right visual field (RVF), extending horizontally from fixation to a position $4.0 \mathrm{~cm}\left(4.0^{\circ}\right.$ of visual angle) to the left or right of fixation (see Figure 1).

The comparison figures were of a single type consisting of two parallel horizontal lines, each $2.0 \mathrm{~cm}$ long and $0.04 \mathrm{~cm}$ thick (see Figure 1). There were 66 different comparison-figure heights, ranging from $0.70 \mathrm{~cm}$ to $5.32 \mathrm{~cm}$ as required by the procedure described below. Each comparison figure was centered vertically on fixation and appeared in either the LVF or the RVF, extending horizontally between positions 1.0 and $3.0 \mathrm{~cm}\left(1.0^{\circ}\right.$ and $\left.3.0^{\circ}\right)$ to one side of fixation (as shown in Figure 1).

Design and Procedure. Following initial screening, instructions, and 17 practice trials, each subject performed 240 trials in six blocks of 40 trials, with a $40-\mathrm{sec}$ break between blocks. The order of the trials was randomized separately for each subject. In each trial, a test figure was presented to the subject in either the LVF or the RVF for $100 \mathrm{msec}$, followed after $1,900 \mathrm{msec}$ by a comparison figure presented in either the LVF or the RVF for $100 \mathrm{msec}$. The signal tone and fixation cross were presented $500 \mathrm{msec}$ before the onset of each figure, and the cross was removed $100 \mathrm{msec}$ before the appearance of the figure.

The subjects were instructed to estimate the height of the first (test) figure and to remember it until the second (comparison) figure appeared, and then to respond as quickly as possible by pressing the $S$ key if the second figure appeared shorter in height than the first figure, or the L key if the second figure appeared longer in height than the first figure. The subjects were instructed to be prepared to respond with either hand, but when the comparison figure appeared, to respond using only the index finger of the hand on the same side as the comparison-figure presentation. After the response (or after $3 \mathrm{sec}$ if there was no response), a delay of between 2 and $3 \mathrm{sec}$ elapsed (on a random basis) before the fixation cross for the next trial appeared.

Each subject performed 20 trials in each of the 12 conditions resulting from the combination of test-figure type $(0,4,9)$, visual field of the test figure (LVF, RVF), and visual field of the comparison figure (LVF, RVF). In the data analysis, however, the six conditions with test and comparison figures in opposite visual fields (crossed conditions) were excluded, leaving six valid (uncrossed) conditions representing the combination of two within-subject independent variables: test-figure type $(0,4,9)$ and visual field of presentation (LVF, RVF). The 20 trials in each condition consisted of all 10 test-figure heights (from 0.88 to $4.84 \mathrm{~cm}$ in $0.44-\mathrm{cm}$ increments), each paired with two comparison-figure heights. In order for the procedure to yield accurate estimates of perceived test-figure heights, it was necessary to choose these two comparison-figure heights so that one was a little less than the mean perceived testfigure height and one was a little greater than the mean perceived test-figure height. On the basis of pilot work with the 0,4 , and 9 figures, the following comparison-figure heights were selected 
to fulfill these criteria: For the 0 test figure, one comparison figure was $20 \%$ shorter than the test figure and one comparison figure was the same height as the test figure, whereas for the 4 and 9 test figures, one comparison figure was $10 \%$ shorter than the test figure and one comparison figure was $10 \%$ longer than the test figure. Note that because of the effect of the illusion, it was necessary to use different comparison-figure heights for the 0 figure than for the 4 and 9 figures. Unpublished work has shown, however, that when the comparison-figure heights are the same for all of the test figures, the magnitude of the illusion is roughly the same as that obtained in the present study. (Note also that the pilot work indicated that the 0 figure would be perceived to be about $10 \%$ shorter than its true height; this effect will be discussed more fully in the Results and Discussion section for this experiment.)

The primary dependent variable was the perceived height of the test figure, expressed as a percentage increase over the true height. This measure was calculated as follows. For each of the six conditions for a subject, the $S$ and $L$ responses were collapsed across the 10 figure heights, yielding two proportions ( $\mathrm{L}$ responses $\div$ total responses) for the two relative heights of the comparison figures. The psychophysical method of constant stimuli (Finney, 1971; Gescheider, 1985, pp. 38-46) was then used to convert these two proportions to a measure of the perceived height of the test figure. In terms of this method, the test figure was the standard stimulus, the comparison figure was the variable stimulus, and the dependent variable was the height of the variable stimulus at the point of subjective equality (PSE), expressed as a percentage increase over the true height of the standard stimulus.

In addition to this primary dependent variable, two measures of task performance were calculated. First, the just noticeable difference (JND) was obtained from the method of constant stimuli. In this context, the JND measured how well a subject was able to perform discriminations of figure height. Finally, mean response time (RT) was calculated from the latencies between the onset of the comparison figure and the response.

Because the subjects varied greatly in their ability to perform the required judgments, a criterion for subject exclusion was established prior to the data analysis in order to ensure that only reliable scores were used. This criterion was as follows: If for any of the original 12 conditions for a subject, the proportion of $L$ responses to the longer comparison figure was not greater than the proportion of $L$ responses to the shorter comparison figure by at least .15 , the data for that subject were excluded. This criterion was not intended to reflect a subject's susceptibility to the illusion; rather, its purpose was to eliminate subjects whose perceived heights of the test figures could not be accurately measured, either because they could not make the appropriate judgments and were just guessing, or because their perceived heights were far away from the range of comparison-figure heights that we used. As noted earlier, 9 subjects were excluded by this criterion.

\section{Results and Discussion}

The mean perceived heights of the test figures are shown in Figure 3 for (a) the two visual fields and (b) the two sexes. As expected, the overall mean perceived heights for the 4 figure $(-1.10 \%)$ and the 9 figure $(-2.73 \%)$ were greater than that for the 0 figure $(-10.17 \%)$, indicating an effect of the illusion. The perceived heights were analyzed in a $2 \times 4 \times 3 \times 2$ mixed-design analysis of variance, with sex (female, male) and response-key configuration (SL-SL, SL-LS, LS-SL, LS-LS) as the between-subjects factors, and test-figure type $(0,4,9)$ and visual field of presentation (LVF, RVF) as the within-subjects factors.
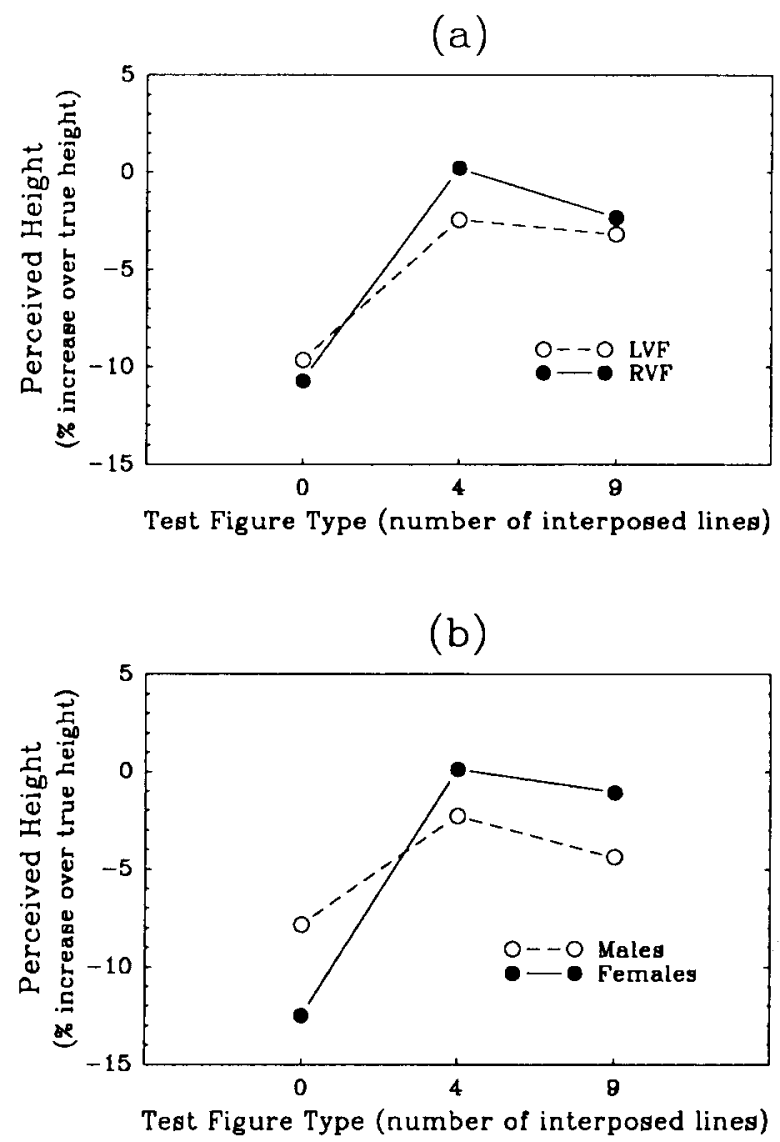

Figure 3. Mean perceived heights of the test figures in Experiment 1 for (a) the two visual fields and (b) the two sexes.

This analysis showed that the main effect of test-figure type was significant $[F(2,16)=42.78, p<.001]$, and that the interaction between test-figure type and sex was also significant $[F(2,16)=8.80, p<.005]$. The interaction between test-figure type and visual field of presentation, however, was not significant $[F(2,16)=1.47$, $p>.05]$, and no other main effects or interactions reached significance at the .05 level.

Illusion magnitude. Post hoc comparisons of the overall means (Tukey test with .05 significance level, critical value of difference $=3.82 \%$ ) showed that the perceived heights of the 4 and 9 figures did not differ significantly from each other, but were both significantly greater than the perceived height of the 0 figure. Thus, an appropriate measure of the illusion magnitude is the contrast that subtracts the perceived height of the 0 figure from the average of the perceived heights of the 4 and 9 figures. Figure 4 shows the means of these illusion magnitudes for (a) the two visual fields and (b) the two sexes. The difference between the illusion magnitudes in the LVF $(6.85 \%)$ and the RVF $(9.67 \%)$ was not significant $[F(1,8)=2.29$, $p=.17]$, but the illusion magnitude for females $(12.04 \%)$ 

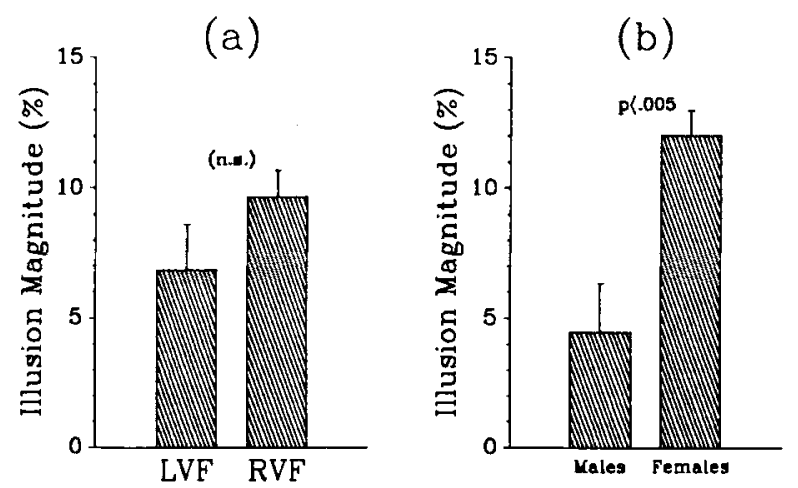

Figure 4. Mean illusion magnitudes in Experiment 1 for (a) the two visual fields and (b) the two sexes. Error bars show 1 standard error.

was significantly greater than for males $(4.48 \%)[F(1,8)=$ $16.31, p<.005]$. Finally, an analysis of simple effects showed that there was not a significant visual field difference in illusion magnitudes for either sex alone $(p s>.05)$.

Because there was not a significant effect of visual field of presentation on illusion magnitude, the existence of a hemispheric difference in susceptibility to the OppelKundt illusion in the successive comparison mode is not supported by this experiment. Furthermore, the measure of the visual field difference in illusion magnitude (LVF - RVF) had a 95\% confidence interval that extended from $-7.11 \%$ to $+1.48 \%$, so this study shows that a substantially greater right hemisphere susceptibility to the Oppel-Kundt illusion in the successive comparison mode is unlikely. It does remain possible, however, that the left hemisphere is more susceptible to the illusion in this mode, and that a more powerful study is required to detect this difference.

The sex difference in susceptibility to the illusion was unexpected, because an extensive study by Porac, Coren, Girgus, and Verde (1979) failed to find such a difference for the Oppel-Kundt illusion (or for nearly all of the $\mathbf{4 5}$ illusion variants tested). In that study, however, the subjects were allowed to inspect the illusion figure freely, and it is possible that the illusion-presentation mode used in the present experiment emphasized additional cognitive processes that are sex-dependent.

Shrinkage effect. It should be noted that judgments of the $\mathbf{0}$ figure height were consistently negative (perceived to be roughly $10 \%$ shorter than the true height), and that the effect of the illusion in the 4 and 9 figures was to counteract this "shrinkage effect," thereby creating judgments close to the true height. It is unlikely that the primary cause of the shrinkage effect was the shorter mean relative height of the comparison figures for the 0 test figure, because pilot work showed that the shrinkage effect was also strong when the mean comparison-figure height was the same as the test-figure height. (In fact, this pilot data was used to select the comparison-figure heights for Experiment 1.) The shrinkage of the perceived extent of a briefly exposed figure when compared to a second figure occurring after a short delay has also been observed for straight lines (Erlebacher \& Sekuler, 1974). Thus, the shrinkage effect seems to reflect a decrease over time of the apparent extent of a figure after it has been removed from view.

JND and RT. Analyses of variance were carried out for the JND data (overall mean $=12.43 \%$ ) and the RT data (overall mean $=967 \mathrm{msec}$ ) in the same design that was used for the perceived heights. For JND, there were no significant main effects or interactions at the .05 level. The RT analysis revealed a marginally significant main effect of figure type $[F(2,16)=3.46, p=.055]$, with faster responses for the 4 figure $(960 \mathrm{msec})$ and the 9 figure ( $955 \mathrm{msec}$ ) than for the 0 figure $(985 \mathrm{msec})$. The only RT effect actually reaching significance at the .05 level was an interaction between figure type and sex $[F(2,16)=5.90, p=.012]$, with the figure-type effect noted above occurring for females but not for males (recall that the illusion magnitudes were also greater for females than for males). It is counterintuitive that responses would be faster for figures that produce the Oppel-Kundt illusion, but similar effects have been observed with the Poggendorff and Ponzo illusions (Clayson, 1978).

\section{EXPERIMENT 2 SIMULTANEOUS COMPARISONS, NORMAL SUBJECTS}

In the second experiment, we used a simultaneous comparison mode, in which subjects were shown a single figure containing both filled and unfilled extents to be compared with each other in a quick judgment. Thus, the illusion-processing time was minimized in this experiment.

In the previous experiment, the subjects were required to determine their response hand on the basis of the visual field of the comparison figure (visual cuing). In Experiment 2, a tactile cuing method was also used. Subjects in the tactile cuing group were cued by the stimulation of the same finger that was to be used for the response, whereas subjects in the visual cuing group determined their response hand as before. It was hypothesized that tactile cuing would make the determination of response hand more automatic, thus creating less interference and perhaps a more spontaneous response from the hemisphere being tested. Therefore, it was predicted that subjects using tactile cuing would have lower JNDs and faster RTs than subjects using visual cuing.

\section{Method}

Subjects. The subjects were 20 undergraduate students $(11 \mathrm{fe}-$ males and 9 males) who had not participated in Experiment 1, but who had the same characteristics as the subjects used in that study. Eleven additional subjects participated but were excluded because their data were unreliable (see Design and Procedure subsection). Random assignment created visual cuing and tactile cuing groups with 9 and 11 valid subjects, respectively. These subjects were 18-28 years of age, with a mean age of 19 years, 5 months.

Apparatus. The apparatus was the same as that used in Experiment 1 , except that all responses were collected from two specially designed response boxes, one for each hand (see Figure 5). Each response box contained a vertical shaft upon which the subjects 


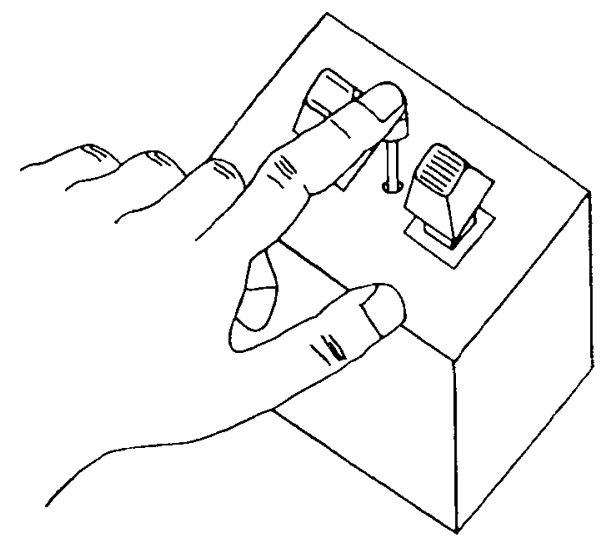

Figure 5. Response box used in Experiment 2. (Two boxes were used, one for each hand.) The shaft that the finger rests on may be lifted $0.10 \mathrm{~cm}$ by a solenoid contained within the box to cue the subject for a response from that hand.

rested their index fingers. This shaft was part of a $12-\mathrm{V}$ dc continuous-duty push-type solenoid (Guardian TP 8X16-C-12D) that could lift the shaft (and finger) a distance of $0.10 \mathrm{~cm}$ with $0.8 \mathrm{~kg}$ of force. Also on each box were two keys, positioned $2.0 \mathrm{~cm}$ to the left and to the right of the shaft, which were labeled with symbols showing unfilled and filled extents (U key and F key, respectively). The labeling order of these keys was randomized for each subject.

Stimuli. The stimuli consisted of the same fixation cross used in Experiment 1, and three types of stimulus figures (see Figure 2). Each figure consisted of six horizontal, parallel, and evenly spaced lines, with a seventh horizontal, parallel line placed well above the other six lines, thus forming an unfilled extent positioned above (and joined to) a filled extent with four interposed lines. All of the lines were $2.0 \mathrm{~cm}$ long and $0.04 \mathrm{~cm}$ (1 pixel) thick. The three types of figures differed in the relative heights of the unfilled and filled extents; these types were labeled $20 \%, 30 \%$, and $40 \%$ (representing the amounts by which the unfilled extents were greater than the filled extents). Finally, for each of the three types of figures, there were three absolute heights of the filled extents $(1.32,1.76$, and $2.20 \mathrm{~cm}$ ). The figures were positioned vertically so that the filled extents were below fixation and the empty extents were above fixation. Each figure appeared in either the LVF or the RVF, extending horizontally between positions 1.0 and $3.0 \mathrm{~cm}\left(1.0^{\circ}\right.$ and $3.0^{\circ}$ ) to one side of fixation (as shown for the $40 \%$ figure in Figure 2).

Design and Procedure. The subjects were given practice and were tested in blocks of trials as in Experiment 1. In each trial, a single stimulus figure was presented in either the LVF or the RVF for $100 \mathrm{msec}$; the fixation cross (without a tone) preceded the figure by $900 \mathrm{msec}$ and was removed $100 \mathrm{msec}$ before the appearance of the figure. For the tactile cuing group, when the stimulus figure appeared, the solenoid for the hand ipsilateral to the figure was turned on, so that the index finger of that hand (resting on the shaft of the solenoid) was cued by being lifted $0.10 \mathrm{~cm}$. The solenoids were not turned on for the visual cuing group.

The subjects were instructed that for each trial, they were to decide which of the two extents in the figure appeared greater in height, and then to respond as quickly as possible by pressing the key with the symbol showing the greater extent (U key or F key). All of the subjects responded only with the index finger of the hand ipsilateral to the stimulus presentation. The tactile cuing group, however, was not instructed to pay attention to the visual field of presentation, but was told only to respond with the hand that was cued with the tactile stimulus. The visual cuing group was instructed as in Experiment 1. After the response (or after $3 \mathrm{sec}$ if there was no response), the solenoid shaft returned to its original position (tactile cuing group only), and $2 \mathrm{sec}$ elapsed before the start of the next trial.

Each subject performed a total of 324 trials (in six blocks of 54); there were 18 repeated trials (three in each block) for each combination of visual field of presentation (LVF, RVF), stimulus-figure type $(20 \%, 30 \%, 40 \%)$, and filled-extent height $(1.32 \mathrm{~cm}, 1.76 \mathrm{~cm}$, $2.20 \mathrm{~cm}$ ). For each subject, the responses for these trials were collapsed across the three filled-extent heights, and proportions (U responses $\div$ total responses) were calculated for the three stimulusfigure types in each visual field. The method of constant stimuli was then applied to these three proportions to find the PSE between the unfilled and filled extents. This PSE measures the percentage effect of the illusion, or illusion magnitude (the primary dependent variable).

As in Experiment 1, the JND and mean RT (latency between onset of stimulus figure and response) were also calculated and used as dependent measures. In addition, the standard error for each illusionmagnitude measurement was determined using the formula suggested by Finney (1971, pp. 33-34, Equation 3.12) and verified through a random sampling experiment by Masin and Vidotto (1982). The standard error of the difference between the illusion magnitudes in the two visual fields was then calculated for each subject, and if this standard error exceeded a value of $6.00 \%$, the data for that subject were excluded on the basis of poor reliability. (This criterion was established prior to the examination or analysis of the illusion-magnitude data.)

\section{Results and Discussion}

As Figure 6 shows, the mean illusion magnitude was greater in the LVF (36.98\%) than in the RVF (32.38\%). The illusion magnitudes were analyzed in a $2 \times 2 \times 2$ mixed-design analysis of variance with unequal groups (unweighted means). Cuing method (visual, tactile) and sex (female, male) were the two between-subjects factors, and visual field of presentation (LVF, RVF) was the within-subjects factor. The analysis showed that the main effect of visual field of presentation was significant $[F(1,16)=8.78, p<.01]$. No other main effects or interactions reached significance at the .05 level.

Because LVF presentations of the stimulus figure were always accompanied by left-hand responses, and RVF presentations were always accompanied by right-hand responses, the main effect of visual field on illusion magnitude implies that the subjects' right hemispheres were more susceptible than their left hemispheres to the Oppel-

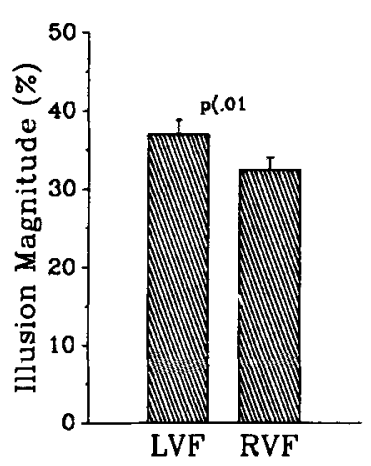

Figure 6. Mean illusion magnitudes in Experiment 2 for the two visual fields. Error bars show 1 standard error. 
Kundt illusion in the simultaneous comparison mode. In order to determine the significance of effects within individual subjects, for each subject the difference between the illusion magnitudes in the two visual fields was divided by the standard error of this difference and compared to a $t$ distribution with 10 degrees of freedom (the worst case for the approximate degrees of freedom calculated for each subject according to a formula derived by Rothwell, 1988). This analysis showed that at a significance level of .05 (two-tailed), 8 of the 20 subjects showed greater illusion magnitudes in the LVF, but only 1 subject showed a greater illusion magnitude in the RVF.

Analyses of variance for the JND data (overall mean = $15.63 \%$ ) and the RT data (overall mean $=951 \mathrm{msec}$ ) in the same design that was used for the illusion-magnitude data revealed no significant main effects or interactions for these measures at the .05 level. Because there was no effect of cuing method on either JND or RT, the hypothesis that tactile cuing would make the judgment task easier was not supported. However, an effect of cuing method on these performance measures could have been masked to some degree by the exclusion of subjects that did not perform above criterion. In fact, a greater proportion of subjects were excluded from the visual cuing group ( 8 of 17) than from the tactile cuing group ( 3 of 14); but this difference is not significant in Fisher's exact test (twotailed $p=.26$ ).

\section{EXPERIMENT 3 SIMULTANEOUS COMPARISONS, SPLIT-BRAIN PATIENT}

In the previous experiment, the visual field difference in illusion magnitude, although significant, was not large when compared to the total illusion magnitude. Such a result might be expected for normal subjects, who may transfer visual information across the cerebral commissures and then be influenced by the processing of both hemispheres in each trial. Thus, in an attempt to discover the susceptibilities of the truly isolated hemispheres in a single case (and to replicate the finding of the previous experiment), a commissurotomy patient was tested with the Oppel-Kundt illusion in Experiment 3, again using a simultaneous comparison mode. On the basis of the results of Experiment 2 and the results of previous testing of the same patient with the Müller-Lyer illusion (Kumar et al., 1976), it was predicted that the illusion magnitude would be greater in the LVF than in the RVF.

\section{Method}

Subject. Commissurotomy Patient L.B. (age 36) served as the single subject in two sessions of testing. L.B. underwent complete surgical transection of the corpus callosum, anterior commissure, and hippocampal commissure by P. J. Vogel and J. E. Bogen at the age of 12 years, 10 months to alleviate intractable epilepsy. He is right-handed, with dominance for language in the left hemisphere, and he is believed to have minimal extracallosal brain damage (for the case history and a review of the disconnection syndrome, see Bogen \& Vogel, 1975; Sperry, Gazzaniga, \& Bogen, 1969). L.B.'s visual acuity (with corrective glasses) is normal in his right eye; during testing, he wore his glasses and his left eye was covered.

Apparatus and Stimuli. The apparatus was the same as that used in Experiment 2. In the first session of testing, the order of the response keys (from left to right as viewed by the subject) was $U$, $F$ for the left hand and $F, U$ for the right hand. In the second session, the response boxes were rotated $90^{\circ}$ so that both $\mathrm{F}$ keys were on the bottom and both $U$ keys were on the top (making the configuration compatible with the stimulus figures). The stimuli were the same as in Experiment 2, except that there were six stimulus figure types $(10 \%, 20 \%, 30 \%, 40 \%, 50 \%, 60 \%)$, and only two filled-extent heights $(1.76 \mathrm{~cm}, 2.20 \mathrm{~cm})$.

Design and Procedure. L.B. was tested in two sessions 28 days apart. The individual trials in these sessions were identical to those in Experiment 2, except that the stimulus figure was presented for $140 \mathrm{msec}$ rather than for $100 \mathrm{msec}$, and up to $5 \mathrm{sec}$ was allowed for a response. All of the trials used tactile cuing for response hand (solenoids activated).

L.B. performed 576 trials in the first session and 1,152 trials in the second session; these trials were equally distributed across visual field of presentation (LVF, RVF), stimulus-figure type (10\%, 20\%, $30 \%, 40 \%, 50 \%, 60 \%)$, and filled-extent height $(1.76 \mathrm{~cm}, 2.20 \mathrm{~cm})$. As in Experiment 2, the responses were collapsed across the filledextent heights, and the method of constant stimuli was used to calculate the illusion magnitudes in the two visual fields. Standard errors, JNDs, and mean RTs were also calculated as in the previous experiment.

\section{Results and Discussion}

The magnitudes of the illusion in the two visual fields are shown in Figure 7. Contrary to the prediction, in both sessions L.B. showed a greater illusion effect in the RVF (Session 1, 47.06\%; Session 2, 39.77\%) than in the LVF (Session 1, 35.52\%; Session 2, 34.65\%). For each session, the difference between the illusion magnitudes in the two visual fields was divided by the standard error of this difference to obtain a $t$ statistic with degrees of freedom given approximately by the formula derived by Rothwell (1988). This analysis showed that the results were significant for both Session $1[t(50)=2.87, p<.01]$ and Session $2[t(196)=3.54, p<.001]$. Thus, it may be concluded that L.B.'s left hemisphere was more susceptible than his right hemisphere to the Oppel-Kundt illu-
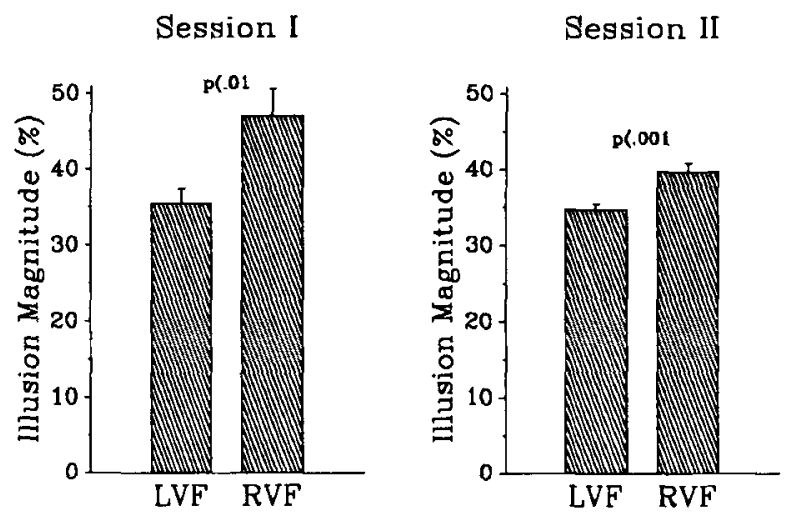

Figure 7. Illusion magnitudes for Subject L.B. (Experiment 3) for the two visual fields in Sessions 1 and 2. Error bars show 1 standard error, calculated as described in the text. 
sion in the simultaneous comparison mode. It should be noted that although the prediction of a greater right hemisphere susceptibility was based in part on a previous study with the same patient (Kumar et al., 1976), this previous study used a different illusion (Müller-Lyer) under different viewing conditions (free vision), and these differences could be responsible for the different results.

In the first session, L.B. was tired from having been tested in other experiments for several hours, and this fact is reflected by relatively poor task-performance measures (mean JND $=30.89 \%$, mean RT $=1,573 \mathrm{msec}$ ). In the second session he was alert, and his performance was better (mean JND $=15.71 \%$, mean RT $=1,333 \mathrm{msec}$ ). In both sessions, however, the JND was lower in the LVF (Session 1, 23.26\%; Session 2, 12.05\%) than in the RVF (Session 1, 38.52\%; Session 2, 19.36\%), indicating that L.B.'s right hemisphere was better than his left hemisphere at making height discriminations. (This visual field difference in JND was consistent in magnitude across both halves of Session 1 and across all four quarters of Session 2.) This result supports the accepted idea that the right hemisphere is specialized for visuospatial tasks, but it should be noted that the left hemisphere was able to perform the task at an acceptable level for the statistical analysis. Furthermore, the mean RTs were nearly identical for the two visual fields in both sessions.

\section{GENERAL DISCUSSION}

The results of the first two experiments show that hemispheric differences in susceptibility to the Oppel-Kundt illusion depend on the temporal parameters of the illusion presentation. These experiments indicate that the right hemisphere is more susceptible in the simultaneous comparison mode, but that it is not more susceptible in the successive comparison mode; in fact, the left hemisphere may be more susceptible in this mode. These results are in agreement with the findings of Clem and Pollack (1975) for the Müller-Lyer illusion (as discussed in the introduction). In our third experiment, however, the simultaneous comparison mode resulted in a greater left hemisphere susceptibility in the commissurotomy patient L.B. This result, which was unexpected in light of the previous findings, presents the challenge of providing a common explanation for the results of all three experiments.

\section{A Preliminary Hypothesis}

A speculative response to this challenge follows a closer examination of the data. First, note that although Experiments 2 and 3 used nearly identical procedures, the mean RTs in Experiment 3 (1,573 and 1,333 msec for Sessions 1 and 2, respectively) were much longer than in Experiment $2(951 \mathrm{msec})$. Furthermore, the difference between the first two experiments may also be viewed in terms of the timing of the response if one considers the critical factor to be the time between the initial illusion presentation and the response (which we refer to as the illusionprocessing time), because this interval included a 2-sec delay in Experiment 1 (for the successive comparison) that was not present in Experiment 2. Thus, the results of all three experiments are consistent with the hypothesis that the illusion-processing time plays a critical role in determining which hemisphere is more susceptible to the illusion, with an increase in processing time associated with a shift of greater susceptibility away from the right hemisphere. (It is worth noting that the only subject in Experiment 2 who had a significantly greater left hemisphere susceptibility also had a relatively long mean RT: 1,265 msec.)

\section{Bin Analysis of RT}

A prediction that follows from this hypothesis is that within each experiment, the difference between the illusion magnitudes in the two visual fields (LVF - RVF) was greater for faster responses than for slower responses. This prediction was tested by a post hoc examination of the data. For each experiment, the responses were collapsed across all subjects (or, in Experiment 3, across both sessions) and then divided into bins according to the RTs. The method of constant stimuli was then applied to the responses within each bin to obtain a measure of the illusion magnitudes in the two visual fields.

Figure 8 shows the results of the RT bin analyses for all three experiments. These results confirm the prediction: For each experiment, the analysis revealed an interaction between RT and visual field of presentation, with an increase in RT generally associated with a decrease in illusion magnitude in the LVF, but not in the RVF. A linear-regression analysis (using two-tailed $p$ s) showed that the regression lines plotted for the LVF and RVF points in Figure $\mathbf{8}$ had significantly different slopes for Experiment $1[t(2)=4.53, p<.05]$, Experiment $2[t(10)=$ $3.03, p<.05]$, and Experiment $3[t(6)=3.49, p<.05]$. Furthermore, the slopes of the LVF regression lines were significantly less than zero for Experiment $2[t(5)=8.44$, $p<.001]$ and Experiment $3[t(3)=4.98, p=.016]$, but the difference for Experiment 1 did not reach significance $[t(1)=2.79, p=.22]$. None of the RVF regressionline slopes differed from zero at a significance level of .05 , although the slope for Experiment 2 was almost significantly less than zero $[t(5)=2.449, p=.058]$.

\section{A Dual-Process Theory}

These findings, as well as the overall results of the three experiments, are consistent with the hypothesis that two separate processes, each available to both hemispheres, are normally involved in the perception and analysis of the Oppel-Kundt illusion figure. The first process is fast, and it results in the right hemisphere being more susceptible to the illusion immediately after its presentation. The second process is slow, and it results in a decline over time of the susceptibility of the right hemisphere, but not of the left hemisphere. Thus, in Experiment 2 (Figure 8b), when quick responses were made, only the fast process was used, so the right hemisphere was more susceptible to the illusion. When the responses were made more slowly, however, both processes were used, so the susceptibilities were more nearly equal. (The results of Ex- 
(a)

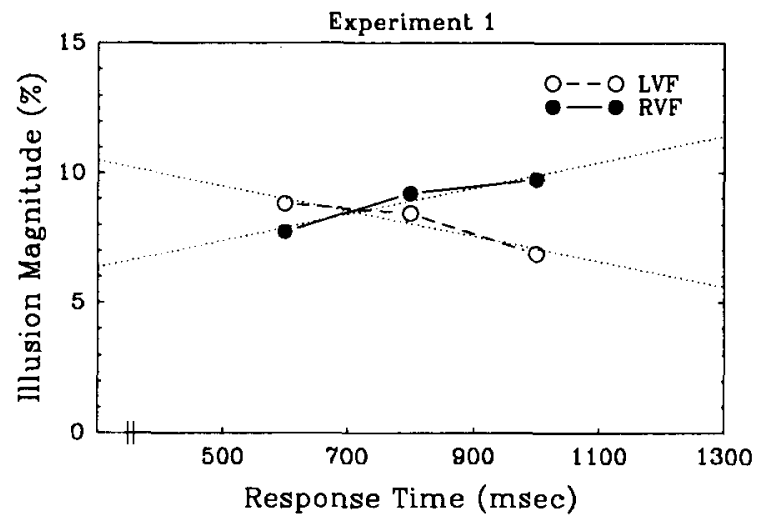

(b)

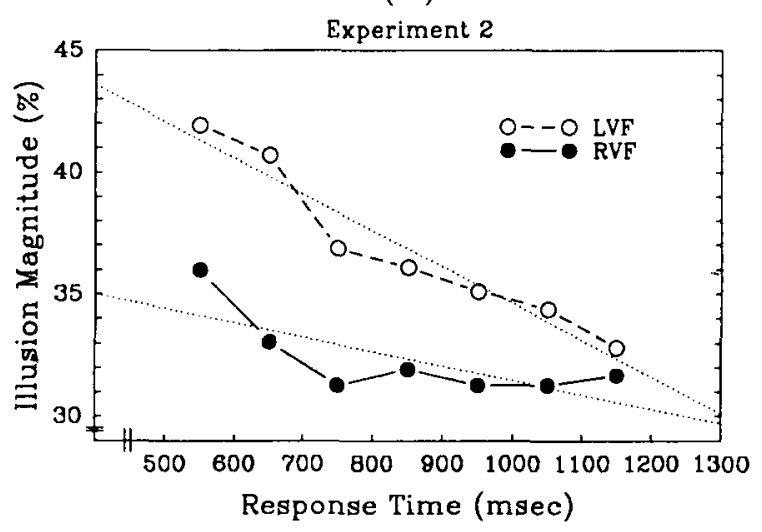

(c)

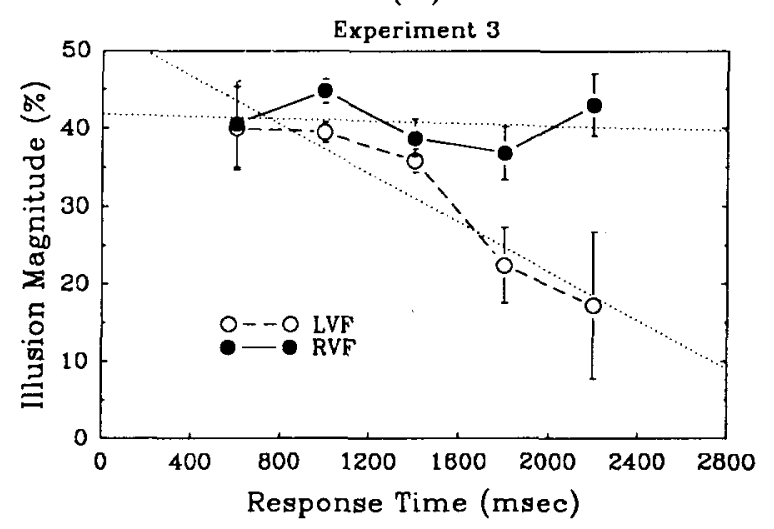

Figure 8. Results of response-time bin analyses for Experiments 1, 2 , and 3. Separate regression lines (dotted) are shown for the two visual fields in each experiment. Error bars in (c) show \pm 1 standard error, calculated as before. Standard errors were calculated only for Experiment 3 because the collapse across subjects in Experiments 1 and 2 would have created an overestimation of the variability. The bin widths (200 msec, 100 msec, and $400 \mathrm{msec}$ for Experiments 1,2 , and 3 , respectively) were based on the total number of responses available in each experiment; in Experiments 1 and 2 these bins were used only if they contained more than 300 responses, and in Experiment 3 they were used only if they resulted in measurements with standard errors both less than $10 \%$. periments 1 and 3 were somewhat different and will be discussed below.) Overall, the combination of the two processes in each hemisphere resulted in the observed shift of greater susceptibility away from the right hemisphere as the processing time increased.

There is increasing evidence that cognitive processes that appear serial from a logical point of view are actually implemented in the brain as parallel computations, and that this is true for each hemisphere as it processes the information independently of the other in the normal brain. For example, the two decisions in binary choice tasks may reflect two separate and parallel processes even though they form logical complements of each other and a decision about one of them could completely determine the other. Consider the paradoxical finding that same decisions are faster than different decisions in pattern-pair comparison tasks. It has been suggested that two separate processes are involved in the comparison: a fast, holistic process for same judgments and a slower, analytic process for different judgments (Bamber, 1969; Farell, 1985). Furthermore, lateralized same-different comparison tasks often show an RVF superiority for same decisions but an LVF superiority for different decisions (e.g., Egeth \& Epstein, 1972). Similarly, Zaidel, Clarke, and Suyenobu (in press) argue that lateralized lexical decision tasks can be processed in either hemisphere and involve separate and parallel word-detection and nonword-detection processes with relative timings that are different in the two hemispheres.

Feature extraction versus visuospatial analysis. It is worthwhile to speculate about the nature of the fast and slow processes proposed as an account of the present results. According to our favored interpretation, the faster process relies on feature extraction for the perception of the illusion figure. The left hemisphere uses a local feature analysis, whereas the right hemisphere uses a more holistic, global feature analysis (Bradshaw \& Nettleton, 1981); thus, the faster process results in a greater right hemisphere susceptibility to the illusion (because the right hemisphere is less able to ignore the distracting features that create the illusion). The slower process, however, relies on visuospatial analysis for computing distances between parts of the illusion figure. The right hemisphere is superior at this analysis (Bradshaw \& Nettleton, 1983) and computes a more accurate representation of the figure (Kosslyn, 1987); thus, as the processing time increases and the slower process becomes more fully expressed, the susceptibility of the right hemisphere decreases more than that of the left hemisphere. Perhaps the right hemisphere is superior at this later stage because it is better able to maintain and operate on the memory representation of an image (as suggested by Moscovitch, Scullion, \& Christie, 1976).

This interpretation of the fast and slow processes resolves the two conflicting predictions described in the introduction by suggesting that these predictions are valid on different time scales. That is, the first prediction (greater right hemisphere susceptibility due to field- 
dependence) is true immediately following the illusion presentation, but the second prediction (lesser right hemisphere susceptibility due to superior visuospatial skills) describes what happens when additional processing time is allowed.

As noted above, the results of Experiments 1 and 3 vary somewhat from what would be expected according to the two-process hypothesis. However, the results of Experiment 3 (Figure 8c) can be accounted for by the claim that the commissurotomy patient L.B. never used the fast process to analyze the illusion figure. Thus, as the processing time increased, his right hemisphere became less susceptible than his left hemisphere. In Experiment 1, it must be assumed that the RT reflects the time used for the comparison judgment, rather than for the processing of the original test stimulus. Thus, it is not clear that the RT bin analysis should have produced the same pattern of results that it did for Experiment 2. Nevertheless, the data shown in Figure 8a indicate that something like the slow process (but not the fast process) may have occurred during the comparison judgment. This result makes sense, because the process of comparing the simple two-line figure to an image in memory probably would not rely as heavily on feature extraction as it would on visuospatial analysis.

Hemispheric interaction? An alternative interpretation of the slow process should be considered. In Experiment 2 , the susceptibilities of the two hemispheres converged to similar values after roughly $1,200 \mathrm{msec}$ (see Figure 8b), so it could be hypothesized that the slow process responsible for this convergence is hemispheric interaction rather than differential visuospatial analysis. In other words, commissural transfer of a representation of the test figure and interhemispheric communication during the processing of that figure could have allowed the two hemispheres to converge upon a single perceived height of the test stimulus (given sufficient processing time), regardless of the visual field of presentation. Hemispheric interaction could also explain the nearly equal overall susceptibilities of the hemispheres in Experiment 1 , because this experiment always used a long test-comparison interval during which the interaction could have taken place. The hemispheric interaction hypothesis, however, cannot explain the decreasés of right hemisphere susceptibility in Experiments 1 and 3, because as Figures $8 \mathrm{a}$ and $8 \mathrm{c}$ show, these decreases both created a divergence of the perceptions by the two hemispheres. In any case, it is not likely that the hemispheres of the commissurotomy patient used in Experiment 3 were able to interact substantially.

\section{Future Work and Final Conclusions}

Future research is required to determine the natures of the fast and slow processes. If, for example, it could be shown that following the presentation of the illusion to normal subjects in the simultaneous mode, the susceptibility of the right hemisphere declined from a level greater than that of the left hemisphere to a level less than that of the left hemisphere, then the hemispheric interaction hypothesis would be invalidated as an interpretation of the slow process (because in this example, the slow process would be leading to diverging perceptions at long processing times). Also, in order to support a causal relationship between the illusion-processing time and hemispheric differences in susceptibility, the present findings should be replicated in a study in which the illusionprocessing time is directly manipulated within a single experiment. Perhaps this manipulation could be achieved through the variation of the test-comparison interval, or through some other means of delaying the generation of a response.

If future studies use the method of constant stimuli, they should aim to maximize the sensitivity of this method. A problem with the present experiments was that a great deal of concentration by the subjects was required to produce a sensitive measure of the illusion magnitude. Because many members of the undergraduate subject pool could not be sufficiently motivated to provide such an effort, a number of these subjects had to be excluded (as noted previously). By increasing the number of trials and keeping the number of conditions to a minimum, future studies could avoid this problem.

It is important to ask why Experiment 2 was able to demonstrate a significant visual field difference in illusion magnitude for normal subjects, whereas most previous such attempts have failed. It may be that the Oppel-Kundt illusion, which has not been used in previously published studies of visual field differences, is more suitable than other illusions for creating a hemispheric difference in susceptibility, because this illusion probably stresses a strong central processing component (i.e., differential attention to the two extents) that is more likely to show hemispheric differentiation.

Finally, we must ask what the present results regarding the processing of the Oppel-Kundt illusion tell us about the nature of visual information processing in general. According to the favored interpretation, these findings support the view that during the analysis of visual information by the brain, at least two separate processes are initiated independently: one process is fast, and it uses feature extraction, whereas a second, slower process uses visuospatial analysis to compute distances between parts of visual stimuli. Furthermore, the present evidence suggests that the faster process is performed more effectively in the left hemisphere, but that the slower process is performed more effectively in the right hemisphere.

\section{REFERENCES}

BAMBER, D. (1969). Reaction times and error rates for "same"'"different" judgments of multidimensional stimuli. Perception \& Psychophysics, 6, 169-174.

Basso, A., Bisiach, E. , Faglioni, P. (1974). The Mueller-Lyer illusion in patients with unilateral brain damage. Cortex, 10, 26-35. Bertelson, P., Morais, J. (1983). A Ponzo-like illusion left and right of fixation: A failed prediction. Neuropsychologia, 21, 105-109. 
Bogen, J. E., \& Vogel, P. J. (1975). Neurologic status in the long term following complete cerebral commissurotomy. In F. Michel \& B. Schott (Eds.), Les syndromes de disconnexion calleuse chez lhomme (pp. 227-251). Lyon, France: Hôpital Neurologique.

Bradshaw, J. L., \& Nettleton, N. C. (1981). The nature of hemispheric specialization in man. Behavioral \& Brain Sciences, 4, 51-91.

Bradshaw, J. L., Nettleton, N. C. (1983). Human cerebral asymmetry. Englewood Cliffs, NJ: Prentice-Hall

BRYDEN, M. P. (1977). Measuring handedness with questionnaires. Neuropsychologia, 15, 617-624.

Clayson, D. E. (1978). Reaction time, bilateral differences, and the Poggendorff and Ponzo illusions. Perceptual \& Motor Skills, 47, 871-874.

Clem, R. K. , \& Pollack, R. H. (1975). Illusion magnitude as a function of visual field exposure. Perception \& Psychophysics, 17, 450-454.

Cohen, B. D., Berent, S., \& Silverman, A. J. (1973). Fielddependence and lateralization of function in the human brain. Archives of General Psychiatry, 28, 165-167.

COREN, S., G GIRGUS, J. S. (1978). Seeing is deceiving: The psychology of visual illusions. Hillsdale, NJ: Erlbaum.

COREN, S., \& HOENIG, P. (1972). Eye movements and decrement in the Oppel-Kundt illusion. Perception \& Psychophysics, 12, 224-225.

CoRen, S., \& PORAC, C. (1987). Individual differences in visualgeometric illusions: Predictions from measures of spatial cognitive abilities. Perception \& Psychophysics, 41, 211-219.

Cronin-Golomb, A. (1986). Figure-background perception in right and left hemispheres of human commissurotomy subjects. Perception, 15, 95-109.

DAviDOFF, J. (1982). Studies with non-verbal stimuli. In J. G. Beaumont (Ed.), Divided visual field studies of cerebral organisation (pp. 2955). London: Academic Press.

EGETH, H., \& EPSTEIN, J. (1972). Differential specialization of the cerebral hemispheres for the perception of sameness and difference. Perception \& Psychophysics, 12, 218-220.

Erlebacher, A., \& Sexuler, R. (1974). Perceived length depends on exposure duration: Straight lines and Müller-Lyer stimuli. Journal of Experimental Psychology, 103, 724-728.

FARELL, B. (1985). "Same"'-"different"' judgments: A review of current controversies in perceptual comparisons. Psychological Bulletin, 98, 419-456.

FinNeY, D. J. (1971). Probit analysis (3rd ed.). Cambridge: Cambridge University Press.

Gescheider, G. A. (1985). Psychophysics: Method, theory, and application (2nd ed.). Hillsdale, NJ: Erlbaum.

GiRgus, J. S., \& CoRen, S. (1973). Peripheral and central components in the formation of visual illusions. American Journal of Optometry \& Archives of American Academy of Optometry, 50, 533-540.

Greist, S. M., \& Grier, J. B. (1977). The effect of retinal location on the magnitude of the Poggendorff illusion. Perception \& Psychophysics, 21, 249-252.

Houlard, N., Fraisse, P., \& HeCAEN, H. (1976). Effects of unilateral hemispheric lesions on two types of optico-geometric illusions. Cortex, 12, 232-240.

KossLYN, S. M. (1987). Seeing and imagining in the cerebral hemispheres: A computational approach. Psychological Review, 94, 148-175.

Kumar, S., \& Bogen, J. E. (1974). Does the Müller-Lyer illusion have lateralizing significance? In Abstracts of the Society for Neuroscience, 4th Annual Meeting (p. 295). Washington, DC: Society for Neuroscience.

Kumar, S., Zaidel, E., \& Bogen, J. E. (1976). Müller-Lyer illusion in disconnected right and left cerebral hemispheres [Abstract]. $A b$ stracts of the Society for Neuroscience, 2, 1081.

Magnussen, S., LANDRo, N. I., \& Johnsen, T. (1985). Visual halffield symmetry in orientation perception. Perception, 14, 265-273.

Masin, S. C., \& VidotTo, G. (1982). A review of the formulas for the standard error of a threshold from the method of constant stimuli. Perception \& Psychophysics, 31, 585-588.

Moscovitch, M., Scullion, D., \& Christie, D. (1976). Early versus late stages of processing and their relation to functional hemispheric asymmetries in face recognition. Journal of Experimental Psychology: Human Perception \& Performance, 2, 401-416.

OLDFIELD, R. C. (1971). The assessment and analysis of handedness: The Edinburgh inventory. Neuropsychologia, 9, 97-113.

Piaget, J. (1969). The mechanisms of perception (G. N. Seagrim, Trans.). London: Routledge \& Kegan Paul. (Original work published 1961)

Porac, C., Coren, S., Girgus, J. S., \& Verde, M. (1979). Visualgeometric illusions: Unisex phenomena. Perception, 8, 401-412.

Robinson, J. O. (1972). The psychology of visual illusion. London: Hutchinson.

ROTHWELL, B. (1988). A derivation of the approximate degrees of freedom for inferential statistics from the method of constant stimuli. Unpublished manuscript, University of California, Los Angeles.

Sperry, R. W., Gazzaniga, M. S., \& Bogen, J. E. (1969). Interhemispheric relationships: The neocortical commissures; syndromes of hemisphere disconnection. In P. J. Vinken \& G. W. Bruyn (Eds.), Handbook of clinical neurology (Vol. 4, pp. 273-290). Amsterdam: North Holland.

ZAIDEL, E. (1973). Linguistic competence and related functions in the right cerebral hemisphere of man following commissurotomy and hemispherectomy. Dissertation Abstracts International, 34, $2350 \mathrm{~B}$. (University Microfilms No. 73-26, 481)

ZAIDEL, E. (1978). Concepts of cerebral dominance in the split brain. In P. Buser \& A. Rougeul-Buser (Eds.), Cerebral correlates of conscious experience (pp. 263-284). Amsterdam: Elsevier.

Zaidel, E., Clarke, J. M., \& SuYenobu, B. (in press). Hemispheric independence: A paradigm case for cognitive neuroscience. In A. Scheibel \& A. Wechsler (Eds.), Neurobiology of higher cognitive functioning. New York: Guilford.

(Manuscript received February 21, 1989; revision accepted for publication September $8,1989$. 